M. Nakai

Nagoya Math. J.

Vol. 70 (1978), 25-40

\title{
AN EXAMPLE ON CANONICAL ISOMORPHISM
}

\author{
MITSURU NAKAI
}

A nonnegative locally Hölder continuous second order differential $P=P(z) d x d y(z=x+i y)$ on a Riemann surface $R$ is referred to as a density on $R$. A density $P$ is said to be finite if $P$ is integrable over $R$, i.e.

$$
\int_{R} P(z) d x d y<\infty
$$

Suppose that $R$ is hyperbolic, i.e. there exists the harmonic Green's function $G(z, \zeta)$ on $R$. A density $P$ on such a surface $R$ is said to be Green energy finite provided the Green energy integral

$$
\iint_{R \times R} G(z, \zeta) P(z) P(\zeta) d x d y d \xi d \eta<\infty \quad(\zeta=\xi+i \eta)
$$

Using a density $P$ on a Riemann surface $R$ we can consider a second order selfadjoint elliptic differential equation

$$
\Delta u(z)=P(z) u(z) \quad \text { (i.e. } d * d u=u P)
$$

invariantly defined on $R$. Denote by $P(R)$ the space of $C^{2}$ solutions of (3) on $R$ and by $P X(R)$ the space of $u \in P(R)$ with a certain boundedness condition $X$. As for $X$ we take $B$ to mean the boundedness, $D$ the finiteness of the Dirichlet integral

$$
D_{R}(u)=\int_{R} d u \wedge * d u,
$$

$E$ the finiteness of the energy integral with respect to $P$ :

$$
E_{R}(u)=\int_{R}\left(d u \wedge * d u+u^{2} P\right)
$$

and the combinations $B D$ and $B E$ with obvious meanings. We use the standard notations $H(R)$ and $H X(R)$ for $P(R)$ and $P X(R)$ with $P \equiv 0$,

Received March 17, 1975. 
and in this case of harmonic function, $E=D$.

The space $P X(R)(X=B, D, E, B D, B E)$ consists of only constants for nonhyperbolic $R$, and avoiding such a trivial case we assume that $R$ is hyperbolic. Then the operator $T$ defined by

$$
T u=u+\frac{1}{2 \pi} \int_{R} G(\cdot, \zeta) P(\zeta) u(\zeta) d \xi d \eta
$$

is an injective positive linear operator from $P X(R)$ to $H X(P)$ for $X=$ $B, D, E, B D, B E$ (cf. e.g. [1]). We denote by $T_{X}$ the operator $T$ considered only on $P X(R)$, i.e.

$$
T_{X}=T \mid P X(R),
$$

and if $T_{X}: P X(R) \rightarrow H X(R)$ is surjective, then we say that $P X(R)$ is canonically isomorphic to $H X(R)$. For a systematic exposition on canonical isomorphisms we refer to [2].

It is known (cf. e.g. [1]) that: 1) The space $P B D(R)(P B E(R)$, resp.) is dense in $P D(R)$ ( $P E(R)$, resp.) with respect to the topology defined by the uniform convergence on each compact set of $R$ and by the Dirichlet (energy, resp.) integral over $R ; 2$ ) If $P$ is a Green energy finite (finite, resp.) density on $R$, then $T_{B D}\left(T_{B E}\right.$, resp.) is surjective. In view of these there naturally arises the question: Is $T_{D}\left(T_{E}\right.$, resp.) surjective for any Green energy finite (finite, resp.) density $P$ on $R$ ? The purpose of this paper is to answer negatively to this question by proving the following

THEOREM. There exists a both finite and Green energy finite density $P$ on the hyperbolic simply connected Riemann surface $R$ such that $T_{D}$ and $T_{E}$ are not surjective.

It has been known that there exists a density (a finite density, resp.) on the hyperbolic simply connected Riemann surface $R$ such that $T_{B D}$ $\left(T_{B E}\right.$, resp.) is surjective and yet $T_{D}\left(T_{E}\right.$, resp.) is not (Singer [6] ([3], resp.)). Our theorem contains the above as a special case. The simply connectedness of $R$ in our theorem is not an essential restriction. Actually our theorem is true for any Riemann surface with the property $H D(R)$ $-H B D(R) \neq \phi$. The only reason we put the restriction is to simplify the reasoning and to avoid inessential complications. At the end of the introduction the author should mention his indebtness to Professor Moses Glasner who gave him an important incentive to the present work. 
1. As a conformal representation of the hyperbolic simply connected Riemann surface $R$ we take the unit disk in the complex plane $C$. Thus we always mean in the sequel by $R$ the unit disk $|z|<1$ and by $\beta$ the unit circle $|z|=1$. Then a density $P$ may be considered as a nonnegative locally Hölder continuous function $P(z)$ on $R$. The $P$-unit $e$ is defined by

$$
e(z)=\lim _{r \rightarrow 1} e_{r}(z)
$$

where $e_{r}$ is the solution of (3) on the disk $|z|<r<1$ with boundary values 1 on $|z|=r$.

We state a sufficient condition for a given positive bounded solution $u$ of (3) on $R$ to be the $P$-unit $e:$ If

$$
\lim _{r \rightarrow 1} u\left(r \mathrm{e}^{i \theta}\right)=1
$$

for almost every $e^{i \theta} \in \beta$, then $u$ is the $P$-unit $e$. The harmonic Green's function $G(z, \zeta)$ on $R$ is given by

$$
G(z, \zeta)=\log \left|\frac{1-\bar{\zeta} z}{z-\zeta}\right|
$$

and, by (4), the function

$$
T u(z)=u(z)+\frac{1}{2 \pi} \int_{R} \log \left|\frac{1-\bar{\zeta} z}{z-\zeta}\right| d \mu(\zeta)
$$

belongs to $H B(R)$, where $d \mu(\zeta)=P(\zeta) u(\zeta) d \xi d \eta$ is a measure on $R$ with a finite total mass. By the Littlewood theorem (cf. e.g. Tsuji [7])

$$
\lim _{r \rightarrow 1} \int_{R} \log \left|\frac{1-\bar{\zeta} r \mathrm{e}^{i \theta}}{r \mathrm{e}^{i \theta}-\zeta}\right| d \mu(\zeta)=0
$$

for almost every $e^{i \theta} \in \beta$. Therefore (5) implies that $\lim _{r \rightarrow 1} T u\left(r e^{i \theta}\right)=1$ for almost every $e^{i \theta} \in \beta$, and a fortiori the Fatou theorem implies that $T u=1$. In particular, $u \leq 1$ on $R$. By the maximum principle, $u \leq$ $e_{r} \leq 1$ on $|z|<r$ for every $r \in(0,1)$. Hence we conclude that $u \leq e \leq 1$ on $R$ and the condition (5) is also satisfied by $e$. By the same reasoning as above we obtain $T e=1$, i.e. $T u=T e$. The injectiveness of $T$ implies that $u=e$.

A key lemma for the proof of our theorem is the following ingenious result of Singer [5]: For every $u \in P D(R)$ we have

$$
D_{R}\left(e T_{D} u\right)<\infty
$$


Our program of the proof is to find an $h \in H D(R)$ and a positive $C^{\infty}$ subharmonic function $e$ on $R$ with (5) such that $P(z)=\Delta e(z) / e(z)$ satisfies (1) and (2) and yet $D_{R}(e \cdot h)=\infty$. Then $e$ is the $P$-unit and $h \notin T_{D}(P D(R))$ by (6), i.e. $T_{D}$ is not surjective, from which nonsurjectiveness of $T_{E}$ follows since $P E(R) \subset P D(R)$.

2. We start with constructing an $h \in H D(R)$. We use the notation $U(\zeta, r)$ to denote the open disk in $C$ with center $\zeta \in C$ and radius $r>0$. Let $\left\{d_{n}\right\}(n=0,1, \ldots)$ be the sequence determined by

$$
d_{0}=1, \quad d_{n} / d_{n-1}=\exp \left(-2 \pi n^{4}\right) \quad(n=1,2, \cdots) .
$$

We define an $\left.f_{n} \in C(C) \cap H\left(U\left(1, d_{n-1}\right)-\overline{U\left(1, d_{n}\right.}\right)\right)$ by $f_{n}=0$ on $C-U\left(1, d_{n-1}\right)$ and $f_{n}=1$ on $U\left(1, d_{n}\right)(n=1,2, \cdots)$. Then

$$
D_{c}\left(f_{n}\right)=2 \pi / \log \left(d_{n-1} / d_{n}\right)=n^{-4} .
$$

Let $h_{n}=H_{f_{n}}^{R}$, the harmonic function on $R$ with boundary values $f_{n}$ on $\beta$. Clearly $h_{n}>0$ on $R$. By the Dirichlet principle

$$
D_{R}\left(h_{n}\right) \leq D_{R}\left(f_{n}\right)<n^{-4} .
$$

By the triangle inequality

$$
D_{R}\left(\sum_{n=m+1}^{m+p} h_{n}\right) \leq\left(\sum_{n=m+1}^{m+p} n^{-2}\right)^{2}
$$

for every $m$ and $p=1,2, \cdots$. Hence the sequence $\left\{\sum_{n=1}^{m} h_{n}\right\}(m=1,2, \cdots)$ in $H D(R)$ is convergent in the Dirichlet integral on $R$. On the other hand $\sum_{n=1}^{m} h_{n}=0$ on the part $\beta^{-}$of $\beta$ in the second and third quadrants. Therefore

$$
h(z)=\sum_{n=1}^{\infty} h_{n}(z)
$$

is convergent on $R, h \in H D(R) \cap C(\bar{R}-\{1\})$, and $h(z) \rightarrow \infty$ as $z \in \bar{R}-\{1\}$ tends to $z=1$.

We denote by $A_{n}$ the part of $\left.\beta \cap\left[U\left(1, d_{n-1}\right)-\overline{U\left(1, d_{n}\right.}\right)\right]$ in the first quadrant. Then $A_{n}$ is an open arc in $\beta$ such that

$$
h \mid A_{n}>n \quad(n=1,2, \cdots) .
$$

We denote by $a_{n}$ the midpoint of $A_{n}$ and by $2 \sigma_{n}$ the length of $A_{n}$. The sequence $\left\{a_{n}\right\}(n=1,2, \ldots)$ of points in $\beta$ and $\left\{\sigma_{n}\right\}(n=1,2, \ldots)$ of 
positive numbers will be used later in the construction of a subharmonic function $e$ on $R$.

3. The construction of $e$ is rather complicated and requires a bit delicate estimates. Therefore it is convenient to prepare several elementary lemmas first in nos. 3-7, and then the construction will be carried over in nos. 8-11. The proof of the theorem will be completed in the final no. 12 .

Let $\lambda$ be a cross cut in $R$, i.e. an analytic arc contained in $R$ except end points joining two distinct points in $\beta$. We denote by $F$ one of regions in $C$ bounded by $\beta$ and $\lambda$. We take one more cross cut $\gamma$ in $F$ joining two distinct points in $\beta \cap \bar{F}$ different from end points of $\lambda$. The region in $F$ bounded by $\beta$ and $\gamma$ is denoted by $V$. Let $Y$ be the empty set $\phi$ or the union of disks $X_{j}(j=1, \cdots, k)$ such that $\bar{X}_{j} \subset V$ and $\bar{X}_{i}$ $\cap \bar{X}_{j}=\phi(i \neq j)$. Let $\mathscr{F}=\mathscr{F}(F ; Y)$ be the class of functions $u$ in $C(\bar{F}) \cap H(F-\bar{Y})$ such that $u \mid \bar{Y} \cup(\beta \cap \bar{F})=0$. The assertion is: There exists a positive constant $c_{1}=c_{1}(F ; Y, \gamma)$ such that

$$
\sqrt{D_{V}(u)} \leq c_{1} \max _{\lambda}|u|
$$

for every $u \in \mathscr{F}$.

To show this let $\beta_{0}$ be a Jordan arc joining two end points of $\lambda$ outside $\bar{F}$ such that the region $F_{0}$ bounded by $\lambda$ and $\beta_{0}$ contains $F$ and let $Y_{0}$ be the union of disks $X_{j 0}(j=1, \cdots, k)$ concentric to $X_{j}$ such that $\bar{X}_{j 0} \subset X_{j}$. Since $u \mid(\partial(F-\bar{Y})-\lambda)=0$ for every $u \in \mathscr{F}$ which is harmonic on $F-\bar{Y}$, the symmetry principle on harmonic functions assures that every $u \mid(F-\bar{Y})$ simultaneously has the harmonic extension $\tilde{u}$ to $F_{0}-\bar{Y}_{0}$ if we take $\beta_{0}$ and $Y_{0}$ close enough to $\beta$ and $Y$, respectively. By the maximum principle, $|u| \leq \max _{\lambda}|u|$ on $\bar{F}$ and thus

$$
|\tilde{u}| \leq \max _{\lambda}|u|
$$

on $F_{0}-\bar{Y}_{0}$ for every $u \in \mathscr{F}$. Let $a \in \bar{V}-Y, r(a)>0$ be such that $\overline{U(a, 2 r(a))} \subset F_{0}-\bar{Y}_{0}$, and $P\left(z, 2 r(a) e^{i \theta}\right)$ be the Poisson kernel on $U(a, 2 r(a))$, i.e.

$$
v(z)=\int_{0}^{2 \pi} P\left(z, 2 r(a) e^{i \theta}\right) v\left(a+2 r(a) e^{i \theta}\right) d \theta
$$

for every $v$ harmonic on $\overline{U(a, 2 r(a))}$. Since 


$$
\frac{\partial}{\partial t} v(z)=\int_{0}^{2 \pi} \frac{\partial}{\partial t} P\left(z, 2 r(a) e^{i \theta}\right) v\left(a+2 r(a) e^{i \theta}\right) d \theta
$$

for $t=x$ and $y$, we have

$$
\left|\frac{\partial}{\partial t} v(z)\right| \leq K(a) \sup _{|z-a|=2 r(a)}|v|
$$

where

$$
K(a)=\sum_{t=x, y} \sup _{|\zeta-a|=2 r(a),|z-a| \leq r(a)}\left|\frac{\partial}{\partial t} P(z, \zeta)\right|
$$

which is seen to be finite by using the concrete representation of $P(z, \zeta)$. Since $\bar{V}-Y$ is compact, it can be covered by a finite number of disks $V\left(a_{\nu}, r\left(a_{\nu}\right)\right)\left(a_{\nu} \in \bar{V}-Y ; \nu=1, \cdots, \ell\right)$. Then the required $c_{1}^{2}$ is $\left(\max _{1 \leq \nu \leq \ell} K\left(a_{\nu}\right)\right)^{2}$ multiplied by the area of $F-\bar{Y}$.

4. Let $\lambda, \gamma, V$, and $F$ be as in no. 3. This time we assume $Y \neq \phi$, i.e. $Y$ is the union of disks $X_{j}(j=1, \cdots, k)$ such that $\bar{X}_{j} \subset V$ and $\bar{X}_{i} \cap \bar{X}_{j}=\phi(i \neq j)$. By exactly the same consideration as in no. 3, we obtain: There exists a positive constant $c_{2}=c_{2}(F ; Y, \gamma)$ such that

$$
\sum_{j=1}^{k}\left|\int_{\partial Y_{j}} * d u\right| \leq c_{2} \max _{\lambda}|u|
$$

for every $u \in \mathscr{F}=\mathscr{F}(F ; Y)$ and

$$
\left|\int_{r} f * d u\right| \leq c_{2}\left(\max _{r}|f|\right)\left(\max _{\lambda}|u|\right)
$$

for every $f \in C(\gamma)$ and every $u \in \mathscr{F}=\mathscr{F}(F ; Y)$.

5. We introduce the notation $V(\zeta, r)$ for $\zeta \in \beta$ and $r>0$ to mean $V(\zeta, r)=R \cap U(\zeta, r)$. Let $Y$ be the empty set $\phi$ or the union of disks $X_{j}(j=1, \cdots, k)$ such that $\bar{X}_{j} \subset R$ and $\bar{X}_{i} \cap \bar{X}_{j}=\phi(i \neq j)$. Let $\sigma=$ $\sigma(1, Y)$ be the distance dis $(Y, 1)$ between the point $z=1$ and $Y$ if $Y \neq \phi$ and $\sigma=2$ if $Y=\phi$. We fix $Y$ and take one more variable disk $X$ such that $\bar{X} \subset R-\bar{Y}$. Consider the harmonic measure $w=w(\cdot, Y \cup X)$ of $Y \cup X$ in $R$, i.e. $w \in C(\bar{R})$ such that $w \mid \overline{X \cup Y}=1$ and $w \mid \beta=0$. The third lemma is: For any numbers $\eta_{1}, \eta_{2}$ and $s$ with $0<\eta_{1}<\eta_{2}$ and $0<s<\sigma(1, Y)$ there exists a number $\rho \in(0, s)$ and a disk $X$ with $\bar{X} \subset V(1, \rho)$ such that

$$
\eta_{1}<\int_{-\partial X} * d w(\cdot, Y \cup X)<\eta_{2}
$$


where $\partial X$ is positively oriented with respect to $X$, and

$$
\eta_{1}<D_{V(1, \rho)}(w(\cdot, Y \cup X))<\eta_{2} .
$$

Actually we can choose $U(t, \varepsilon)$ as $X$ where $t$ is on the real axis.

6. To prove the assertion in no. 5 we first consider the function

$$
f(t, \varepsilon)=\int_{-\partial U(t, \varepsilon)} * d w(\cdot, Y \cup U(t, \varepsilon))
$$

for $t \in\left[\sigma_{0}, 1\right)\left(\sigma<\sigma_{0}<1\right)$ and $\varepsilon \in(0,1-t)$. As auxiliary results to prove (11) and (12) we assert the following:

$$
\lim _{\varepsilon \rightarrow 0} f\left(\sigma_{0}, \varepsilon\right)=0 ;
$$

For any fixed $\varepsilon_{0} \in\left(0, \min \left[\left(\sigma_{0}-\sigma\right), 1-\sigma_{0}\right]\right)$

$$
f\left(\cdot, \varepsilon_{0}\right) \in C\left[\sigma_{0}, 1-\varepsilon_{0}\right),
$$

i.e. $f\left(t, \varepsilon_{0}\right)$ is continuous on the interval $\left[\sigma_{0}, 1-\varepsilon_{0}\right)$ as a function of $t$, and

$$
\lim _{t \rightarrow 1-\varepsilon_{0}} f\left(t, \varepsilon_{0}\right)=\infty .
$$

We fix an $\varepsilon_{1} \in\left(0, \min \left[\sigma_{0}-\sigma, 1-\sigma_{0}\right]\right)$. Then $w\left(\cdot, Y \cup U\left(\sigma_{0}, \varepsilon\right)\right)$ together with its first derivatives converge to $w(\cdot, Y)$ and its first derivative uniformly on each compact subset of $R-\bar{Y}-\left\{\sigma_{0}\right\}$, and in particular on $\partial U\left(\sigma_{0}, \varepsilon_{1}\right)$, and therefore

$$
\lim _{\varepsilon \rightarrow 0} \int_{\partial U\left(\sigma_{0}, \varepsilon_{1}\right)} * d w\left(\cdot, X \cup U\left(\sigma_{0}, \varepsilon\right)\right)=\int_{\partial U\left(\sigma_{0}, \varepsilon_{1}\right)} * d w(\cdot, X)=0 .
$$

Since $f\left(\sigma_{0}, \varepsilon\right)=-\int_{\partial U\left(\sigma_{0}, \varepsilon_{1}\right)} * d w\left(\cdot, X \cup U\left(\sigma_{0}, \varepsilon\right)\right)$ for $\varepsilon \in\left(0, \varepsilon_{1}\right)$, we deduce (13).

Fix a $t_{0} \in\left[\sigma_{0}, 1-\varepsilon_{0}\right)$ and an $\varepsilon_{1} \in\left(\varepsilon_{0}, \min \left[\sigma_{0}-\sigma, 1-\sigma_{0}\right]\right)$. It is easily seen that $w\left(\cdot, Y \cup U\left(t, \varepsilon_{0}\right)\right)$ together with its first derivatives converge to $w\left(\cdot, Y \cup U\left(t_{0}, \varepsilon_{0}\right)\right)$ and its first derivatives uniformly on each compact subset of $R-Y-\overline{U\left(t_{0}, \varepsilon_{0}\right)}$ and in particular on $\partial U\left(t_{0}, \varepsilon_{1}\right)$ as $t \rightarrow t_{0}$. In view of

$$
f\left(t, \varepsilon_{0}\right)=-\int_{\partial U\left(t_{0}, \varepsilon_{1}\right)} * d w\left(\cdot, Y \cup U\left(t, \varepsilon_{0}\right)\right)
$$

for $t$ in $\left|t-t_{0}\right|<\varepsilon_{1}-\varepsilon_{0}$, we conclude (14) as in the proof of (13).

Let $u_{0}$ be the harmonic measure of $Y \cup U\left(1-\varepsilon_{0}, \varepsilon_{0}\right)$ with respect to 
$R$, i.e. $\left.u_{0} \in C(\bar{R}-\{1\}) \cap H\left(R-\overline{Y \cup U\left(1-\varepsilon_{0}, \varepsilon_{0}\right.}\right)\right)$ such that $u=1$ on $\left.\left.\bar{Y} \cup \overline{\left(U\left(1-\varepsilon_{0}, \varepsilon_{0}\right.\right.}\right)-\{1\}\right)$ and $u=0$ on $\beta-\{1\}$. Suppose $D_{R}\left(u_{0}\right)<+\infty$. By the Dirichlet principle, $D_{R}\left(u_{0}\right)>D_{R}\left(v_{t}\right)$, where $v_{t}=w(\cdot, U(t, \varepsilon))$ with a fixed $\varepsilon \in\left(0, \varepsilon_{0}\right)$ and a $t \in\left(1-\varepsilon_{0}, 1-\varepsilon\right)$. Observe that $D_{R}\left(v_{t}\right)=2 \pi / \log \mu_{t}$ where $\mu_{t}$ is the modulus of the annulus $R-\overline{U(t, \varepsilon)}$. We know that $\lim _{t \rightarrow 1-s} \mu_{t}=1$ (cf. e.g. Sario-Nakai [4; p. 28]), which implies a contradiction. Thus we must have

$$
D_{R}\left(u_{0}\right)=\infty .
$$

On the other hand, $w_{t}=w\left(\cdot, Y \cup U\left(t, \varepsilon_{0}\right)\right)$ together with its first derivatives converge to $u_{0}$ and its first derivatives uniformly on each compact subset of $R-Y-\overline{U\left(1-\varepsilon_{0}, \varepsilon_{0}\right)}$ as $t \rightarrow 1-\varepsilon_{0}$. Therefore

$$
\lim _{t \rightarrow 1-s_{0}}\left|\int_{\partial Y} * d w_{t}\right|=\left|\int_{\partial Y} * d u_{0}\right|<\infty .
$$

By the Fatou lemma, $D_{R}\left(u_{0}\right) \leq \lim _{\inf _{t \rightarrow 1-s_{0}}} D_{R}\left(w_{t}\right)$ and thus

$$
\lim _{t \rightarrow 1-c_{0}} D_{R}\left(w_{t}\right)=\infty \text {. }
$$

Observe that

$$
D_{R}\left(w_{t}\right)=f\left(t, \varepsilon_{0}\right)+\int_{-\partial Y} * d w_{t} .
$$

Hence we see that (15) is valid.

7. We proceed to the proof of the assertion in no. 5. First we choose and fix a $\rho \in(0, s)$ so small that

$$
D_{V(1, \rho)}(w(\cdot, Y))<\left(\eta_{2}-\eta_{1}\right) / 4 .
$$

Let $\gamma=R \cap \partial U(1, \rho)$ and $\lambda=R \cap \partial U(1, \rho / 2)$. The region bounded by $\beta$ and $\gamma(\lambda$, resp.) containg $Y$ in its interior is denoted by $V$ ( $F$, resp.). The above inequality means that

$$
\left|\int_{r} w(\cdot, Y) * d w(\cdot, Y)\right|<\left(\eta_{2}-\eta_{1}\right) / 4 .
$$

We consider auxiliary functions $w_{r} \in C(\bar{R}-\beta \cap \partial U(1, r)) \cap H(R-\bar{Y} \cup \overline{V(1, r)})$ with $w_{r}=1$ on $\bar{Y} \cup[\overline{V(1, r)}-\beta \cap \partial U(1, r)]$ and $w_{r}=0$ on $\beta-\beta \cap \overline{U(1, r)}$ for $r \in(0, \rho / 4)$. For any disk $X$ with $\bar{X} \subset V(1, r)$, the maximum principle yields 


$$
w(\cdot, Y) \leq w(\cdot, Y \cup X) \leq w_{r}
$$

on $R$, and $\sup _{\lambda}|w(\cdot, Y)-w(\cdot, Y \cup X)| \leq \sup _{\lambda}\left|w(\cdot, Y)-w_{r}\right| \equiv \varepsilon(r)$. Since $w_{r}$ converges to $w(\cdot, Y)$ uniformly on each compact of $\bar{R}-\{1\}$, we have $\lim _{r \rightarrow 0} \varepsilon(r)=0$. Observe that

$$
\left|\int_{r} w(\cdot, Y) * d w(\cdot, Y)-\int_{r} w(\cdot, Y \cup X) * d w(\cdot, Y \cup X)\right|
$$

is dominated by the sum of

$$
\sup _{r}|w(\cdot, Y)-w(\cdot, Y \cup X)| \cdot \int_{r}|* d w(\cdot, Y)| \leq \varepsilon(r) \int_{r}|* d w(\cdot, Y)|
$$

and

$$
\left|\int_{r} w(\cdot, Y \cup X) * d(w(\cdot, Y \cup X)-w(\cdot, Y))\right| \cdot
$$

Observe once more that $w(\cdot, Y \cup X)-w(\cdot, Y) \in \mathscr{F}(F ; Y)$ in the sense of no. 4. Thus the last term is dominated by

$$
\begin{aligned}
& c_{2}\left(\sup _{r}|w(\cdot, Y \cup X)|\right)\left(\sup _{r}|w(\cdot, Y \cup X)-w(\cdot, Y)|\right) \\
& \quad \leq c_{2}\left(\max _{r}\left|w_{r}\right|\right) \varepsilon(r) \leq c_{2}\left(\max _{r}|w(\cdot, Y)|+\varepsilon(r)\right) \varepsilon(r)
\end{aligned}
$$

as a consequence of (10). By fixing $r \in(0, \rho / 4)$ so small that

$$
\varepsilon(r) \int_{r}|* d w(\cdot, Y)|+c_{2}\left(\max _{r}|w(\cdot, Y)|+\varepsilon(r)\right) \varepsilon(r)<\left(\eta_{2}-\eta_{1}\right) / 4
$$

we conclude that

$$
\left|\int_{\tau} w(\cdot, Y) * d w(\cdot, Y)-\int_{\tau} w(\cdot, Y \cup X) * d w(\cdot, Y \cup X)\right|<\left(\eta_{2}-\eta_{1}\right) / 4
$$

whenever $\bar{X} \subset U(1, r) \cap R=V(1, r)$. This with (16) gives

$$
\left|\int_{r} w(\cdot, Y \cup X) * d w(\cdot, Y \cup X)\right|<\left(\eta_{2}-\eta_{1}\right) / 2
$$

for every disk $X$ with $\bar{X} \subset V(1, r)$.

We next take $f(t, \varepsilon)$ considered for the present $w(\cdot, Y \cup U(t, \varepsilon)$ ) as in no. 6 for $t \in[r / 2,1)$ and $\varepsilon \in(0,1-t)$. By (13) we can choose $\varepsilon_{0} \in(0, r / 2)$ with $f\left(r / 2, \varepsilon_{0}\right)<\left(\eta_{1}+\eta_{2}\right) / 2$. By (14) and (15), the mean value theorem applied to $f\left(\cdot, \varepsilon_{0}\right) \in C\left[r / 2,1-\varepsilon_{0}\right)$ yields the existence of $t_{0} \in\left(r / 2,1-\varepsilon_{0}\right)$ 
such that $f\left(t_{0}, \varepsilon_{0}\right)=\left(\eta_{1}+\eta_{2}\right) / 2$. Finally we prove that $X=U\left(t_{0}, \varepsilon_{0}\right)$ is the required. First, $\bar{X} \subset U(1, r) \subset U(1, \rho)$. The (11) is clearly satisfied since $\eta_{1}<\left(\eta_{1}+\eta_{2}\right) / 2<\eta_{2}$. Observe that

$$
D_{V(1, \rho)}(w(\cdot, Y \cup X))=f\left(t_{0}, \varepsilon_{0}\right)+\int_{\tau} w(\cdot, Y \cup X) * d w(\cdot, Y \cup X)
$$

where $\gamma$ is oriented in the direction of $\partial V(1, \rho)$. In view of (17) and $f\left(t_{0}, \varepsilon_{0}\right)=\left(\eta_{1}+\eta_{2}\right) / 2$, we conclude that (12) is true.

8. Having finished preparations in nos. $3-7$, we proceed to the construction of $e$ as announced at the end of no. 1. The construction will be carried over related to the $h \in H D(R)$ constructed in no. 2 and in particular related to the sequences $\left\{a_{n}\right\} \subset \beta$ and $\left\{\sigma_{n}\right\}$ defined in no. 2 . First choose an $s_{1} \in\left(0, \sigma_{1}\right)$ such that $h \mid U\left(a_{1}, s_{1}\right)>1$. By no. 5 , we can find a $\rho_{1} \in\left(0, s_{1}\right)$ and a disk $X_{1}$ with $\bar{X}_{1} \subset U\left(a_{1}, \rho_{1}\right)$ such that

$$
\left\{\begin{array}{l}
2^{-1} \cdot 1^{-2}<\int_{-\partial X_{1}} * d w\left(\cdot, X_{1}\right)<2 \cdot 1^{-2} ; \\
2^{-1} \cdot 1^{-2}<D_{V\left(a_{1}, \rho_{1}\right)}\left(w\left(\cdot, X_{1}\right)\right)<2 \cdot 1^{-2}
\end{array}\right.
$$

Next choose $s_{2} \in\left(0, \sigma_{2}\right)$ such that $h \mid U\left(a_{2}, s_{2}\right)>2$. Let $\gamma=\bar{R} \cap \partial U\left(a_{2}, s_{2}\right)$ and $\lambda=\bar{R} \cap \partial U\left(a_{2}, s_{2} / 2\right)$ and $V$ (F, resp.) be the region bounded by $\beta$ and $\gamma\left(\lambda\right.$, resp.) containing $\bar{X}_{1}$. Let $w_{r}$ be the function in the class $C\left(\bar{R}-\beta \cap \partial U\left(a_{2}, r\right)\right) \cap H\left(R-\bar{X}_{1}-\overline{\left.V\left(a_{2}, r\right)\right)}\right.$ such that $w_{r}=1$ on $\bar{X}_{1} \cup$ $\left[\overline{V\left(a_{2}, r\right)}-\beta \cap \partial U\left(a_{2}, r\right)\right]$ and $w_{r}=0$ on $\beta-\overline{U\left(a_{2}, r\right)}$ for $r \in\left(0, s_{2} / 2\right)$. Take any disk $X$ with $\bar{X} \subset U\left(a_{2}, r\right)$. The maximum principle yields $u_{1} \leq u \leq w_{r}$ on $R$ where $u_{1}=w\left(\cdot, X_{1}\right)$ and $u=w\left(\cdot, X_{1} \cup X\right)$. Observe that $u_{1}-u$ $\in \mathscr{F}\left(F, X_{1}\right)$ in the sense of nos. 3 and 4 . Since $\left|u-u_{1}\right| \leq\left|u_{1}-w_{r}\right|$,

$$
\left|\int_{-\partial X_{1}} * d u-\int_{-\partial X_{1}} * d u_{1}\right| \leq c_{2} \max _{\lambda}\left|u_{1}-w_{r}\right|,
$$

where $c_{2}$ is the constant in (9), and

$$
\begin{aligned}
& \left|\sqrt{D_{V\left(a_{1}, \rho_{1}\right)}(u)}-\sqrt{D_{V\left(a_{1}, \rho_{1}\right)}\left(u_{1}\right)}\right| \leq \sqrt{D_{V\left(a_{1}, \rho_{1}\right)}\left(u-u_{1}\right)} \\
& \leq \sqrt{D_{V}\left(u-u_{1}\right)} \leq c_{1} \max _{\lambda}\left|u_{1}-w_{r}\right|
\end{aligned}
$$

where $c_{1}$ is the constant in (8). Since $w_{r}$ converges to $u_{1}$ uniformly on each compact subset of $\bar{R}-\left\{a_{2}\right\}$ as $r \rightarrow 0$, by using (18) and the above we can find an $r_{2} \in\left(0, s_{2} / 2\right)$ such that 


$$
\left\{\begin{array}{l}
2^{-1} \cdot 1^{-2}<\int_{-\partial X_{1}} * d w\left(\cdot, X_{1} \cup X\right)<2 \cdot 1^{-2} \\
2^{-1} \cdot 1^{-2}<D_{V\left(a_{1}, \rho_{1}\right)}\left(w\left(\cdot, X_{1} \cup X\right)\right)<2 \cdot 1^{-2}
\end{array}\right.
$$

for any disk $X$ with $\bar{X} \subset U\left(a_{2}, r_{2}\right)$. Again using the results in no. 5, we can find a $\rho_{2} \in\left(0, r_{2}\right)$ and a disk $X_{2}$ with $\bar{X}_{2} \subset U\left(a_{2}, \rho_{2}\right)$ such that

$$
\left\{\begin{array}{l}
2^{-1} \cdot 2^{-2}<\int_{-\partial X_{2}} * d w\left(\cdot, X_{1} \cup X_{2}\right)<2 \cdot 2^{-2} \\
2^{-1} \cdot 2^{-2}<D_{V\left(a_{2}, \rho_{2}\right)}\left(w\left(\cdot, X_{1} \cup X_{2}\right)\right)<2 \cdot 2^{-2}
\end{array}\right.
$$

Combining this with (19) we have

$$
\begin{cases}2^{-1} \cdot j^{-2}<\int_{-\partial X_{j}} * d w\left(\cdot, X_{1} \cup X_{2}\right)<2 \cdot j^{-2} & (j=1,2) ; \\ 2^{-1} \cdot j^{-2}<D_{V\left(a_{j}, \rho_{j}\right)}\left(w\left(\cdot, X_{1} \cup X_{2}\right)\right)<2 \cdot j^{-2} & (j=1,2) .\end{cases}
$$

9. Repeating the process as in no. 8, we can find a sequence $\left\{\rho_{n}\right\}$ with $\rho_{n} \in\left(0, \sigma_{n}\right)$ and a sequence $\left\{X_{n}\right\}$ of disks $X_{n}$ with $\bar{X}_{n} \subset U\left(a_{n}, \rho_{n}\right)$ $(n=1,2, \cdots)$ such that

$$
\left\{\begin{array}{l}
2^{-1} \cdot j^{-2}<\int_{-\partial X_{j}} * d w\left(\cdot, \bigcup_{k=1}^{n} X_{k}\right)<2 \cdot j^{-2} \quad(j=1, \cdots, n) ; \\
2^{-1} \cdot j^{-2}<D_{V\left(a_{j}, \rho_{j}\right)}\left(w\left(\cdot, \bigcup_{k=1}^{n} X_{k}\right)\right)<2 \cdot j^{-2} \quad(j=1, \cdots, n) ; \\
h \mid V\left(a_{n}, \rho_{n}\right)>n
\end{array}\right.
$$

for every $n=1,2, \ldots$. Although it should be clear by no. 8 , we show how to find $\rho_{n+1}$ and $X_{n+1}$ when $\left\{\rho_{\nu}\right\}(\nu=1, \cdots, n)$ and $\left\{W_{\nu}\right\}(\nu=1, \cdots, n)$ satisfying (21) have already been found. Choose $s_{n+1} \in\left(0, \sigma_{n+1}\right)$ such that $h \mid U\left(a_{n+1}, s_{n+1}\right)>n+1$. Let $\gamma=\bar{R} \cap \partial U\left(a_{n+1}, s_{n+1}\right)$ and $\lambda=\bar{R} \cap \partial U\left(a_{n+1}, s_{n+1} / 2\right)$ and $V(F$, resp.) be the region bounded by $\beta$ and $\gamma(\lambda$, resp.) containing $\bigcup_{k=1}^{n} \bar{X}_{k}$. Let $w_{r}$ be the function in the class

$$
C\left(\bar{R}-\beta \cup \partial U\left(a_{n+1}, r\right)\right) \cap H\left(R-\bigcup_{k=1}^{n} \bar{X}_{k}-\overline{V\left(a_{n}, r\right)}\right)
$$

such that $w_{r}=1$ on $\left(\bigcup_{k=1}^{n} \bar{X}_{k}\right) \cup\left[\overline{V\left(a_{n+1}, r\right)}-\beta \cap \partial U\left(a_{n+1}, r\right)\right]$ and $w_{r}=0$ on $\beta-\overline{U\left(a_{n+1}, r\right)}$ for $r \in\left(0, s_{n+1} / 2\right)$. Take any disk $X$ with $\bar{X} \subset U\left(a_{n+1}, r\right)$. The maximum principle yields

$$
u_{n} \leq u \leq w_{r}
$$

on $R$ where $u_{n}=w\left(\cdot, \bigcup_{k=1}^{n} X_{k}\right)$ and $u=w\left(\cdot,\left(\cup_{k=1}^{n} X_{k}\right) \cup X\right)$. Observe 
that $u-u_{n} \in \mathscr{F}\left(F, \bigcup_{k=1}^{n} X_{k}\right)$ in the sense of nos. 3 and 4 . Since $\left|u-u_{n}\right|$ $\leq u_{n}-w_{r}$,

$$
\sum_{j=1}^{n}\left|\int_{-\partial X_{j}} * d u-\int_{-\partial X_{j}} * d u_{n}\right| \leq c_{2} \max _{\lambda}\left|u_{n}-w_{r}\right|,
$$

where $c_{2}$ is the constant in (9), and

$$
\begin{gathered}
\left|\sqrt{D_{V\left(a_{j}, \rho_{j}\right)}(u)}-\sqrt{D_{V\left(a_{\left.j, \rho_{j}\right)}\right.}\left(u_{n}\right)}\right| \leq \sqrt{D_{V\left(a_{j}, \rho_{j}\right)}\left(u-u_{n}\right)} \\
\leq \sqrt{D_{V}\left(u-u_{n}\right)} \leq c_{1} \max _{\lambda}\left|u_{n}-w_{r}\right|
\end{gathered}
$$

for $j=1,2, \cdots, n$. Since $w_{r}$ converges to $u_{n}$ uniformly on each compact of $\bar{R}-\left\{a_{n+1}\right\}$ as $r \rightarrow 0$, by using (18) and the above, we can find an $r_{n+1}$ $\in\left(0, s_{2} / 2\right)$ such that

$$
\left\{\begin{array}{l}
2^{-1} \cdot j^{-2}<\int_{-\partial X_{j}} * d w\left(\cdot,\left(\bigcup_{k=1}^{n} X_{k}\right) \cup X\right)<2 \cdot j^{-2} \quad(j=1, \cdots, n) ; \\
2^{-1} \cdot j^{-2}<D_{V\left(a_{j}, \rho_{j}\right)}\left(w\left(\cdot,\left(\bigcup_{k=1}^{n} X_{k}\right) \cup X\right)\right)<2 \cdot j^{-2} \quad(j=1, \cdots, n)
\end{array}\right.
$$

for every disk $X$ with $\bar{X} \subset U\left(a_{n+1}, r_{n+1}\right)$. Again using the results in no. 5 we can find a $\rho_{n+1} \in\left(0, r_{n+1}\right)$ and a disk $X_{n+1}$ with $\bar{X}_{n+1} \subset U\left(a_{n+1}, \rho_{n+1}\right)$ such that

$$
\left\{\begin{array}{l}
2^{-1} \cdot(n+1)^{-2}<\int_{-\partial X_{n+1}} * d w\left(\cdot, \bigcup_{k=1}^{n+1} X_{k}\right)<2 \cdot(n+1)^{-2} \\
2^{-1} \cdot(n+1)^{-2}<D_{V\left(a_{n+1}, \rho_{n+1}\right)}\left(w\left(\cdot, \bigcup_{k=1}^{n+1} X_{k}\right)\right)<2 \cdot(n+1)^{-2}
\end{array}\right.
$$

and clearly $h \mid U\left(a_{n+1}, \rho_{n+1}\right)>n+1$. Combining this with (22) for $X=X_{n+1}$, we deduce that $\left\{\rho_{k}\right\}(k=1, \cdots, n+1)$ and $\left\{X_{k}\right\}(k=1, \cdots, n+1)$ satisfies (21).

10. Since $w\left(\cdot, \cup_{k=1}^{n} X_{k}\right)$ increases as $n$ increases and is bounded by 1 ,

$$
w\left(\cdot, \bigcup_{k=1}^{\infty} X_{k}\right)=\lim _{n \rightarrow \infty} w\left(\cdot, \bigcup_{k=1}^{n} X_{k}\right)
$$

exists on $R$, which is continuous on $\bar{R}-\{1\}, 1$ on $\bigcup_{k=1}^{\infty} X_{k}, 0$ on $\beta-\{1\}$, harmonic on $R-\bigcup_{k=1}^{\infty} \bar{X}_{k}$, and superharmonic on $R$. Set $u_{n}=w\left(\cdot, \bigcup_{k=1}^{n} X_{k}\right)$ and $u_{\infty}=w\left(\cdot, \bigcup_{k=1}^{\infty} X_{k}\right)$. Then

$$
D_{R}\left(u_{n+p}-u_{n}\right)=\sum_{k=n+1}^{n+p} \int_{-\partial X_{k}}\left(1-u_{n}\right) * d\left(u_{n+p}-u_{n}\right)
$$




$$
=\sum_{k=n+1}^{n+p}\left(\int_{-\partial X_{k}}\left(1-u_{n}\right) * d u_{n+p}-D_{X_{k}}\left(u_{n}\right)\right) .
$$

Here $0<1-u_{n}<1$ and $* d u_{n+p}>0$ on $-\partial X_{k}$ for $k=n+1, \cdots, n+p$ and a fortiori by (21)

$$
D_{R}\left(u_{n+p}-u_{n}\right) \leq 2 \sum_{k=n+1}^{n+p} k^{-2} .
$$

On letting $p \rightarrow \infty$ and by using the Fatou lemma

$$
D_{R}\left(u_{\infty}-u_{n}\right) \leq 2 \sum_{k=n+1}^{\infty} k^{-2}
$$

for every $n$. Thus

$$
\lim _{n \rightarrow \infty} D_{R}\left(w\left(\cdot, \bigcup_{k=1}^{\infty} X_{k}\right)-w\left(\cdot, \bigcup_{k=1}^{\infty} X_{k}\right)\right)=0 .
$$

On the other hand,

$$
\begin{aligned}
D_{R}\left(w\left(\cdot, \bigcup_{k=1}^{n} X_{k}\right)\right) & =\sum_{k=1}^{n} \int_{-\partial X_{k}} * d w\left(\cdot, \bigcup_{k=1}^{n} X_{k}\right) \\
& \leq 2 \sum_{k=1}^{n} k^{-2}
\end{aligned}
$$

and we conclude with (24) that

$$
D_{R}\left(w\left(\cdot, \bigcup_{k=1}^{\infty} X_{k}\right)\right) \leq 2 \sum_{k=1}^{\infty} k^{-2}<\infty
$$

Passing to the limit in (21) by using (23) and (24) we obtain the following:

$$
\left\{\begin{array}{l}
2^{-1} \cdot j^{-2} \leq \int_{-\partial X_{j}} * d w\left(\cdot, \bigcup_{k=1}^{\infty} X_{k}\right) \leq 2 \cdot j^{-2} \quad(j=1,2, \cdots) ; \\
2^{-1} \cdot j^{-2} \leq D_{V\left(a_{j}, \rho_{j}\right)}\left(w\left(\cdot, \bigcup_{k=1}^{\infty} X_{k}\right)\right) \leq 2 \cdot j^{-2} \quad(j=1,2, \cdots) ; \\
h \mid V\left(a_{j}, \rho_{j}\right)>j \quad(j=1,2, \cdots) .
\end{array}\right.
$$

11. Take two concentric disks $W_{n}$ and $Z_{n}$ to $X_{n}$ such that $\bar{Z}_{n} \subset$ $X_{n} \subset \bar{X}_{n} \subset W_{n} \subset \bar{W}_{n} \subset U\left(a_{n}, \rho_{n}\right) \quad(n=1,2, \cdots)$. By applying the regularization (cf. e.g. Yosida [8], Tsuji [7], Sario-Nakai [4; p. 150]) to $w\left(\cdot, \cup_{n=1}^{\infty} X_{n}\right)$ on each $W_{n}-\bar{Z}_{n}(n=1,2, \cdots)$, the resulting $C^{\infty}$ superharmonic function on $R$ will be denoted by $g$. Then

$$
g \mid\left(R-\bigcup_{n=1}^{\infty}\left(W_{n}-\bar{Z}_{n}\right)\right)=w\left(\cdot, \bigcup_{n=1}^{\infty} X_{k}\right) .
$$


The first inequality of (26) is also valid if the integrating curve $-\partial X_{j}$ is replaced by $-\partial W_{j}$ and a fortiori we have

$$
2^{-1} \cdot j^{-2} \leq \int_{-\partial W_{j}} * d g \leq 2 \cdot j^{-2} \quad(j=1,2, \cdots) .
$$

We can also make $D_{W_{j}-Z_{j}}\left(g-w\left(\cdot, \bigcup_{n=1}^{\infty} X_{n}\right)\right)$ as small as we wish by choosing the regularization $g$ close enough to $w\left(\cdot, \bigcup_{n=1}^{\infty} X_{n}\right.$ ) (cf. e.g. Sario-Nakai $\left[4 ;\right.$ p. 150]) in each $W_{j}-\bar{Z}_{j}(j=1,2, \cdots)$ and thus the second inequality of (26) yields

$$
4^{-1} \cdot j^{-2} \leq D_{V\left(a_{j}, \rho_{j}\right)}(g) \leq 4 \cdot j^{-2} \quad(j=1,2, \cdots)
$$

and we stress here once more the following

$$
h \mid V\left(a_{j}, \rho_{j}\right)>j \quad(j=1,2, \cdots) .
$$

Finally we set $e(z)=1-g(z) / 2$ and observe that

$$
1 / 2 \leq e(z) \leq 1
$$

on $R$ and that $e(z)$ is $C^{\infty}$ subharmonic on $R$. As the counter parts of (27)-(29) we obtain

$$
\left\{\begin{array}{l}
4^{-1} \cdot j^{-2} \leq \int_{\partial W_{j}} * d e \leq j^{-2} \quad(j=1,2, \cdots) \\
16^{-1} \cdot j^{-2} \leq D_{V\left(a_{j}, \rho_{j}\right)}(e) \leq j^{-2} \quad(j=1,2, \cdots) \\
h \mid V\left(a_{j}, \rho_{j}\right)>j \quad(j=1,2, \cdots) .
\end{array}\right.
$$

12. The required density $P$ in the theorem is given by

$$
P(z)=\Delta e(z) / e(z)
$$

on $R$. Then $e$ is a bounded solution of (3) with this $P$. Since $e$ has boundary values 1 on $\beta-\{1\}$, the condition (5) in no. 1 is satisfied by $e$ and therefore $e$ is the $P$-unit for this $P$. Therefore

$$
1=T e=e+\frac{1}{2 \pi} \int_{R} G(\cdot, \zeta) P(\zeta) e(\zeta) d \xi d \eta
$$

where $G(z, \zeta)$ is the harmonic Green's function on $R$.

In view of (30) and (31), we deduce

$$
\begin{aligned}
\int_{R} P(z) d x d y & \leq 2 \int_{R} \Delta e(z) d x d y=2 \sum_{j=1}^{\infty} \int_{W_{j}} \Delta e(z) d x d y \\
& =2 \sum_{j=1}^{\infty} \int_{\partial W_{j}} * d e \leq 2 \sum_{j=1}^{\infty} j^{-2}<\infty,
\end{aligned}
$$


i.e. $P$ is a finite density on $R$. By (30) and (32), we have

$$
\int_{R} G(\cdot, \zeta) P(\zeta) d \xi d \eta \leq 4 \pi
$$

on $R$. Then by the Fubini theorem

$$
\begin{aligned}
\iint_{R \times R} G(z, \zeta) P(z) P(\zeta) d x d y d \xi d \eta & =\int_{R} P(z)\left(\int_{R} G(z, \zeta) P(\zeta) d \xi d \eta\right) d x d y \\
& \leq 4 \pi \int_{R} P(z) d x d y \leq 8 \pi \sum_{j=1}^{\infty} j^{-2}<\infty,
\end{aligned}
$$

i.e. $P$ is a Green energy finite density on $R$.

The last and the most delicate part of the proof of the theorem is to show that $T_{D}$ for the present $P$ is not surjective. For the aim we shall show that the $h$ in $H D(R)$ defined in no. 2 does not have the counter image of $T_{D}$ in $P D(R)$, i.e.

$$
h \notin T_{D}(P D(R)) .
$$

To prove this we estimate $D_{R}(e h)$. Take a concentric disk $\Omega$ to $R$ with $\bar{\Omega} \subset R$. Then

$$
D_{\Omega}(e h)-\int_{\Omega} e^{2} d h \wedge * d h-2 \int_{\Omega} e d h \wedge * h d e=\int_{\Omega} h^{2} d e \wedge * d e
$$

and hence

$$
\int_{\Omega} h^{2} d e \wedge * d e \leq D_{\Omega}(e h)+\int_{\Omega} e^{2} d h \wedge * d h+2\left|\int_{\Omega} e d h \wedge * h d e\right| .
$$

By the Schwarz inequality, the last term is dominated by

$$
2\left(\int_{\Omega} e^{2} d h \wedge * d h\right)^{1 / 2} \cdot\left(\int_{\Omega} h^{2} d e \wedge * d e\right)^{1 / 2} .
$$

In view of $e^{2} \leq 1$, on setting $\ell_{\Omega}=\left(\int_{\Omega} h^{2} d e \wedge * d e\right)^{1 / 2}$, we have

$$
\ell_{\Omega}^{2} \leq D_{\Omega}(e h)+D_{\Omega}(h)+2 D_{\Omega}(h)^{1 / 2} \cdot \ell_{\Omega}
$$

or

$$
\left(\ell_{\Omega}-D_{\Omega}(h)^{1 / 2}\right)^{2} \leq D_{\Omega}(e h)+2 D_{\Omega}(h) .
$$

Since $D_{\Omega}(h)<D_{R}(h)<\infty$, on letting $\Omega \rightarrow R$, we obtain

$$
\left[\left(\int_{R} h^{2} d e \wedge * d e\right)^{1 / 2}-D_{R}(h)^{1 / 2}\right]^{2} \leq D_{R}(e h)+2 D_{R}(h) .
$$


On the other hand, by (29) and (31), we deduce

$$
\begin{aligned}
\int_{R} h^{2} d e \wedge * d e & \geq \sum_{j=1}^{\infty} \int_{U\left(a_{j}, \rho_{j}\right)} h^{2} d e \wedge * d e \\
& >\sum_{j=1}^{\infty} j^{2} D_{U\left(a_{j}, \rho_{j}\right)}(e) \\
& \geq \sum_{j=1}^{\infty} j^{2} \cdot 16^{-1} \cdot j^{-2}=\infty .
\end{aligned}
$$

Therefore, by (33), $D_{R}(e h)=\infty$. By the Singer criterion (6), this means that $h \notin T_{D}(P D(R))$, i.e. $T_{D}$ is not surjective.

The proof of Theorem is herewith complete.

Added in Proof. The author feels it very fortunate that the referee of this paper was at least careful enough to keep the manuscript of this paper safely for almost three years in his drawer without losing it. In the meantime further developements based on this paper have been published by the present author in the following two papers :

1) Extremizations and Dirichlet integrals on Riemann surfaces, J. Math. Soc. Japan, 28 (1976), 581-603;

2) Malformed subregions of Riemann surfaces, J. Math. Soc. Japan, 29 (1977), 779-782.

\section{REFERENCES}

[1] M. Nakai: Dirichlet finite solutions of $\Delta u=P u$ on open Riemann surfaces, Kōdai Math. Sem. Rep., 23 (1971), 385-397.

[2] —-: Order comparisons on canonical isomorphism, Nagoya Math. J., 50 (1973), $67-87$.

[3] _- Canonical isomorphisms of energy finite solutions of $\Delta u=P u$ on open Riemann surfaces, Nagoya Math. J., 56 (1975), 79-84.

[4] L. Sario and M. Nakai: Classification Theory of Riemann Surfaces, Springer, 1970.

[ 5] I. Singer: Dirichlet finite solutions of $\Delta u=P u$, Proc. Amer. Math. Soc., 32 (1972), 464-468.

[6] - Boundary isomorphism between Dirichlet finite solutions of $\Delta u=P u$ and harmonic functions, Nagoya Math. J., 50 (1973), 7-20.

[ 7$]$ M. Tsuji: Potential Theory in Modern Function Theory, Maruzen, 1959.

[ 8] K. Yosida: Functional Analysis, Springer 1965.

Department of Mathematics

Nagoya Institute of Technology

Gokiso, Showa, Nagoya 466, Japan 